\section{Ruxolitinib in combination with prednisone and nilotinib exhibit synergistic effects in human cells lines and primary cells from myeloproliferative neoplasms}

\author{
Alicia Arenas Cortés, ${ }^{1}$ Rosa Ayala Diaz, ${ }^{1}$ Pilar Hernández-Campo, ${ }^{2}$ \\ Julián Gorrochategui, ${ }^{2}$ Daniel Primo, ${ }^{2}$ Alicia Robles, ${ }^{2}$ María Luz Morales, ${ }^{1,3}$ \\ Joan Ballesteros, ${ }^{2}$ Inmaculada Rapado, ${ }^{1}$ Miguel Gallardo, ${ }^{* 3}$ \\ María Linares ${ }^{* 1,3,4}$ and Joaquín Martínez-López $z^{* 1,3,4}$
}

*MG, ML and JM-L contributed equally to this work

${ }^{1}$ Hematology Service, Hospital Universitario 12 de Octubre; ${ }^{2}$ Vivia Biotech, Tres Cantos; ${ }^{3} \mathrm{H} 120-\mathrm{CNIO}$ Haematological Malignancies Clinical Research Unit, Centro Nacional de Investigaciones Oncológicas (CNIO) and ${ }^{4}$ Universidad Complutense de Madrid, Madrid, Spain.

\section{ABSTRACT}

$\mathrm{R}$ uxolitinib is the front-line non-palliative treatment for myelofibrosis (MF). However, a significant number of patients lose or present suboptimal response, are resistant or have unacceptable toxicity. In an attempt to improve response and avoid the adverse effects of this drug, we evaluated the combination of 17 drugs with ruxolitinib in ex vivo models of peripheral blood mononuclear cells from MF patients and cell lines. We found that the combination ruxolitinib and nilotinib had a synergistic effect against $M F$ cells ( $\Delta \mathrm{EC}_{50}$ nilotinib, $-21.6 \%$ ). Moreover, the addition of prednisone to combined ruxolitinib/nilotinib improved the synergistic effect in all MF samples studied. We evaluated the molecular mechanisms of combined ruxolitinib/nilotinib/prednisone and observed inhibition of JAK/STAT (STAT5, 69.2+11.8\% inhibition) and MAPK (ERK, 29.4+4.5\% inhibition) signaling pathways. Furthermore, we found that the triple therapy combination inhibited collagen protein and COL1A1 gene expression in human bone marrow mesenchymal cells. Taken together, we provide evidence that combined ruxolitinib/nilotinib/prednisone is a potential therapy for MF, possibly through the anti-fibrotic effect of nilotinib, the immunomodulatory effect of ruxolitinib and prednisone, and the anti-proliferative effect of ruxolitinib. This combination will be further investigated in a phase Ib/II clinical trial in MF.

\section{Introduction}

Myelofibrosis (MF) is a Philadelphia chromosome-negative chronic myeloproliferative neoplasm (MPN) clinically characterized by stem cell-derived clonal myeloproliferation and a reactive cytokine-driven increase in bone marrow (BM) fibrosis. ${ }^{1,2}$ Patients with MF have a poor prognosis and a median survival of 5.8 years.

Dysregulation of JAK/STAT signaling is the main cause of MPN and, accordingly, inhibitors of the JAK/STAT signal transduction pathway are currently the best clinical approach to treat this disease. Discovered in $2005,{ }^{3-7}$ a mutation in the JAK2 gene resulting in a substitution of valine for phenylalanine (V617F) was found in approximately $90 \%$ of patients with polycythemia vera (PV), and in $50-60 \%$ of patients with essential thrombocythemia (ET) and primary myelofibrosis (PMF). ${ }^{8}$ In addition, mutations in the MPL gene, which encodes the thrombopoietin receptor, were found in approximately $1 \%$ of patients with $\mathrm{MPN},{ }^{9}$ and $12 \%$ of patients with MPN (35-50\% of MF) have mutations in calreticulin (CALR). ${ }^{10-12}$ Interestingly, the mutated forms of CALR acquire the ability to activate the thrombopoietin

Ferrata Storti Foundation

Haematologica 2019

Volume 104(5):937-946

\section{Correspondence:}

MIGUEL GALLARDO

miguelgallardodelgado@gmail.com

Received: July 10, 2018.

Accepted: December 10, 2018.

Pre-published: December 13, 2018.

doi:10.3324/haematol.2018.201038

Check the online version for the most updated information on this article, online supplements, and information on authorship \& disclosures: www.haematologica.org/content/104/5/937

(C)2019 Ferrata Storti Foundation

Material published in Haematologica is covered by copyright. All rights are reserved to the Ferrata Storti Foundation. Use of published material is allowed under the following terms and conditions:

https://creativecommons.org/licenses/by-nc/4.0/legalcode. Copies of published material are allowed for personal or internal use. Sharing published material for non-commercial purposes is subject to the following conditions:

https://creativecommons. org//icenses/by-nc/4.0/legalcode, sect. 3. Reproducing and sharing published material for commercial purposes is not allowed without permission in writing from the publisher. 
receptor and, therefore, constitutively activate the JAK/STAT pathway. ${ }^{13,14}$

Ruxolitinib, a JAK1/JAK2 inhibitor, is the first and only drug approved by the European Medicines Agency for the treatment of PMF, post-PV MF, and post-ET MF15 and is the first-line treatment for MF. Results of the COMFORTI and II clinical trials showed that ruxolitinib produced a reduction in spleen volume, improved MF-related symptoms, and was associated with prolonged overall survival of patients compared with controls. ${ }^{15}$

Despite the beneficial effect of ruxolitinib, a high percentage of patients lose their response at some point during treatment, and others are refractory or present a suboptimal response. Because of this, the use of drug combinations might increase the effectiveness of the treatment and response time, and overcome treatment resistance. Indeed, numerous studies have used this premise, and a number of combinations have been tested in clinical trials with varying success. For instance, whereas the combination of ruxolitinib with simtuzumab (clinicaltrials.gov identifier: 01369498) produced no clinical benefit, ${ }^{16}$ and the combination with lenalidomide (clinicaltrials.gov identifier: 01375140) had to be terminated early because the efficacy objectives were not achieved, ${ }^{17}$ the combination with danazol (clinicaltrials.gov identifier: 01732445) achieved a hematologic stabilization but did not increase the response to ruxolitinib. ${ }^{18}$ Other combinations including ruxolitinib with buparlisib (clinicaltrials.gov identifier: 01730248) or with panobinostat (clinicaltrials.gov identifiers: 01693601 and 01433445 ) are currently under evaluation in clinical trials. In this scenario, the objective of the present study was to develop a drug combination that enhances the effect of ruxolitinib in the treatment of MF.

The proposed combination in this work, ruxolitinib, nilotinib and prednisone, is the result of testing 17 drugs with ruxolitinib to evaluate the best therapy for MF. We hypothesized that this combination would be synergic through a decrease in the proinflammatory status by ruxolitinib and prednisone ${ }^{19}$ and the known antifibrogenic effect of nilotinib, ${ }^{20}$ and would result in a better histological response.

\section{Methods}

\section{Primary samples and cell lines}

Peripheral blood (PB) samples were collected from MF patients and from healthy donors after obtaining informed consent in accordance with the guidelines of the 12 Octubre Hospital ethics committee and the Declaration of Helsinki. The diagnosis of MF was based on 2016 World Health Organization criteria. ${ }^{21} \mathrm{~PB}$ mononuclear cells (PBMCs) were isolated from 6-10 mL of PB by density gradient centrifugation (Ficoll-Paque ${ }^{\mathrm{TM}}$ PLUS, GE Healthcare, Little Chalfont, UK) and were cultured $\left(0.4 \times 10^{6}\right.$ cells/mL) in Methocult TM GF_H4535 supplemented with 20 ng/mL IL-3 and $50 \mathrm{ng} / \mathrm{mL}$ Stem Cell Factor (both from StemCell Technologies, Vancouver, Canada) at $37^{\circ} \mathrm{C}$ in a humidified atmosphere containing $5 \% \mathrm{CO}_{2}$. For the drug screening study, samples from 9 patients were used; age range was 49-83 years, there were 5 males and 4 females, and 6 of them harbored the JAK2-V617F mutation (Online Supplementary Table S1). For synergy studies, all patients (aged 66-83 years) had the JAK2-V617F mutation ( 3 males and 2 females). No patient had been treated previously (Online Supplementary Table S2).

The BA/F3 wild-type (BA/F3 WT), BA/F3 with JAK2-V617F
Table 1. Drugs used in the screening to search for the best combination with ruxolitinib.

\begin{tabular}{lc}
\hline Drug & \multicolumn{1}{c}{ Target } \\
Ruxolitinib & Jak 1/2 inhibitor \\
Nilotinib & PDGF-R. c-kit and BCR/ABL inhibitor \\
\hline Bosutinib & Src/Abl kinase inhibitor \\
Ponatinib & BCR/ABL inhibitor \\
\hline Midostaurin & FLT3 inhibitor \\
Sorafenib & Multikinase inhibitor \\
\hline Buparlisib & PI3K inhibitor \\
Dactolisib & PI3K/Akt/mTOR inhibitor \\
\hline Everolimus & mTOR inhibitor \\
Sonidegib & SMO inhibitor \\
\hline SB 431542 & Inhibitor of TGF- $\beta$ receptor \\
LCL161 & SMAC mimetic \\
\hline Bortezomib & Proteosome inhibitor \\
Panobinostat & Deacetylase histone inhibitor \\
\hline HSP990 & HSP90 inhibitor \\
Prednisone & Immunosuppressant \\
\hline Anagrelide & PDE3 immunosuppressant \\
Danazol & Antigonadotropic and anti-estrogenic activity \\
\hline
\end{tabular}

mutation (BA/F3 V617F JAK2), and WEHI cell lines were kindly provided by Dr Quintás-Cardama (MD Anderson Cancer Center, Houston, TX, USA). The WEHI cell line, which produces IL-3, was cultured in Dulbecco's Modified Eagle's Medium (DMEM) (Biowest, Nuaillé, France) with 10\% heat-inactivated fetal bovine serum (FBS). BA/F3 cells were cultured in Roswell Park Memorial Institute (RPMI) 1640 medium (Biowest, Nuaillé, Francia) with $10 \%$ FBS plus $10 \%$ conditioned medium from WEHI cells. The SET2 cell line (DSMZ, Braunschweig, Germany), which harbors the JAK2-V617F mutation, was cultured in RPMI 1640 with 20\% FBS. The HS27a human BM mesenchymal cell line (DSMZ) was cultured in DMEM with 10\% FBS.

\section{Dose response and synergy analysis}

A total of 10,000-20,000 cells of the different cell lines were seeded per well in 96-well plates in the presence of the drugs alone (Table 1 and Online Supplementary Table S3) or in combination with ruxolitinib. Dimethyl sulfoxide (DMSO) was used as vehicle. After 48-72 hours (h), cell viability was measured by flow cytometry with Annexin V-phycoerythrin (Biolegend, San Diego, CA, USA) or the metabolic WST-8 assay (Cell Counting Kit - 8 BioChemika; Sigma-Aldrich). Drugs were purchased from SigmaAldrich (St. Louis, MO, USA), Tocris (Bristol, UK) or kindly donated by Novartis (Basel, Switzerland).

Peripheral blood mononuclear cells were treated as follows: a) directly delivering the drugs to methylcellulose solid culture at the outset of the experiment (ex vivo, model A); or b) after 14 days of methylcellulose culture (ex vivo, model $\mathrm{B}$ ). In model $\mathrm{B}$, colonyforming cells were collected, washed with phosphate buffer saline (PBS) and cultured in RPMI with 10\% FBS at 15,000 cells per well in 96-well plates in the presence of the drugs alone (Table 1) or in combination with ruxolitinib, for $72 \mathrm{~h}$. DMSO was used as vehicle at a maximum concentration of $0.5 \%$. Flow cytometry to measure myeloid cell viability was performed with monoclonal antibodies against CD45-allophycocyanin-Cy7, CD13-allophycocyanin and Annexin V-phycoerythrin (all from Biolegend, San Diego, CA, USA) using the ExviTech automated flow cytometry plataform. 
Table 2. Results of the dose-response curves of drugs in monotherapy in patients' samples after 72 hours of incubation with drugs: median. First (Q1) and third (Q3) quartiles.

\begin{tabular}{|c|c|c|c|c|c|c|}
\hline Patients' samples & & $E C_{50}(p M)$ & & & $\%$ Sun & \\
\hline Drugs & Median & Q1 & Q3 & Median & Q1 & Q3 \\
\hline Panobinostat & 0.008 & 0.002 & 0.024 & 3.3 & 0.0 & 7.5 \\
\hline Bortezomib & 0.033 & 0.002 & 0.056 & 6.4 & 1.9 & 11.0 \\
\hline Prednisolone & 0.144 & 0.079 & 0.286 & 26.0 & 24.6 & 28.6 \\
\hline HSP990 & 0.041 & 0.029 & 0.048 & 39.9 & 16.6 & 43.7 \\
\hline BKM120 & 0.893 & 0.866 & 0.964 & 28.3 & 25.8 & 34.4 \\
\hline Ponatinib & 1.716 & 1.317 & 3.341 & 0.0 & 0.0 & 0.0 \\
\hline Pomalidomide & 1.961 & 1.961 & 1.961 & 72.1 & 72.1 & 72.1 \\
\hline Anagrelide & 3.518 & 2.528 & 6.787 & 45.8 & 38.3 & 51.7 \\
\hline Bosutinib & 4.517 & 2.674 & 9.740 & 0.0 & 0.0 & 0.0 \\
\hline Nilotinib & 5.996 & 4.804 & 9.651 & 2.5 & 0.0 & 10.3 \\
\hline Danazol & 9.220 & 8.026 & 10.414 & 45.4 & 39.6 & 51.3 \\
\hline Sorafenib & 10.272 & 10.164 & 10.897 & 0.0 & 0.0 & 0.3 \\
\hline Everolimus & 22.606 & 17.676 & 25.872 & 0.0 & 0.0 & 0.0 \\
\hline SB431542 & 27.334 & 16.362 & 38.307 & 0.0 & 0.0 & 0.0 \\
\hline
\end{tabular}

\section{Collagen I expression study}

Hs27a cells were treated with $100 \mathrm{nM}$ ruxolitinib, $1 \mu \mathrm{M}$ nilotinib or $1 \mu \mathrm{M}$ prednisone or their combinations for $1 \mathrm{~h}$. Subsequently, $2 \mathrm{ng} / \mathrm{mL}$ of TGF- $\beta$ (R\&D Systems, Minneapolis, $\mathrm{MN}, \mathrm{USA}$ ) was added and cells were incubated for a further $24 \mathrm{~h}$. Immunocytochemistry and quantitative polymerase chain reaction (qPCR) analysis was used to measure collagen I expression.

\section{Protein array and western blotting}

The effects of $32 \mathrm{nM}$ ruxolitinib, 1.6 $\mu \mathrm{M}$ nilotinib, $0.8 \mu \mathrm{M}$ prednisone and their combinations, on protein phosphorylation were analyzed using the Human Phospho-kinase Array (Proteome ProfilerTM, R\&D Systems) and by western blotting. Antibodies against phosphorylated or non-phosphorylated STAT5 and ERK 1/2 proteins (Cell Signaling Technology, Danvers, MA, USA) were used in western blotting analysis. Tubulin was used as a loading control and was purchased from Abcam (Cambridge Science Park, Cambridge, UK). Proteins were visualized with the ChemiDoc MP imaging system (BioRad laboratories, Hercules, CA, USA), quantified, corrected for housekeeping expression, and normalized to control samples using the ImageLab software program (v.5.1, BioRad).

\section{Quantitative polymerase chain reaction}

Total RNA was prepared with the AllPrep ${ }^{\text {TM }}$ DNA/RNA Micro Kit (Qiagen, Hilden, Germany). Reverse transcription reaction was carried out using the High Capacity cDNA Reverse Transcription Kit system (Life Technologies, Carlsbad, MA, USA). Real-time PCR was performed with Taqman Gene Expression Master Mix and gene-specific Taqman probe COL1A1 (Hs00164004_m1) using the 7900HT Fast Real-Time PCR Systems platform (all from Life Technologies). Normalized gene expression levels were calculated using GAPDH mRNA expression as a housekeeping gene.

\section{Immunocytochemistry}

HS27a cells were fixed with 4\% paraformaldehyde (Merck Millipore, Billerica, MA, USA) permeabilized and blocked with $0.25 \%$ Triton X-100 plus $1 \%$ BSA in PBS for 30 minutes (min). Slides were incubated with antibodies against collagen I (Abcam) for one hour, followed by a 5 -min incubation with $3 \% \mathrm{H}_{2} \mathrm{O}_{2}$ to inactivate endogenous peroxidase. After incubation with a peroxidase-conjugated secondary antibody for $1 \mathrm{~h}$, signals were revealed with 3,3 diaminobenzidine (Abcam). Counterstaining was performed with Carazzi's hematoxylin (AppliChem Panreac, Darmstadt, Germany). Images were visualized on the Eclipse 80i (Nikon) microscope equipped with a DS-Fi1 camera (Nikon, Minato, Tokyo, Japan). Stained areas were calculated with ImageJ (Rasband, W.S., ImageJ, NIH, Bethesda, MD, USA).

\section{Statistical analysis}

The analysis of drug dose-response was performed using the non-linear regression model (Equation 1):

$$
\mathrm{E}=\mathrm{E}_{0}+\mathrm{E}_{\max }-\mathrm{E}_{01}+10\left(\log \mathrm{EC}_{50}-\mathrm{C}\right)
$$

where $\mathrm{C}$ is the drug concentration; $\mathrm{E}$ is the drug effect; Emax, the maximum drug efficacy in terms of survival; $\mathrm{E}_{0}$, survival when only DMSO is applied; $\mathrm{EC}_{50}$, drug concentration in which $50 \%$ of the total drug action is achieved; and $\gamma$ the slope of the curve. The area under curve (AUC) of dose response curves was also calculated.

The study of the behavior of drugs in combination was performed using $\Delta \mathrm{EC}_{50}$, the percentage of difference between $\mathrm{EC}_{50}$ of each drug in combination with ruxolitinib minus their $\mathrm{EC}_{50}$ in monotherapy.

Synergy analysis was performed using Calcusyn v.2.0 (Biosoft, Ferguson, MO, USA). The calculations performed by the program are based on the median-effect equation formulated by Chou. ${ }^{22}$ The combination index (CI) is the parameter by which the synergy or antagonism of two drugs were quantified (Equation 2):

\section{$C I=C D C D|R+C R C R| D$}

where $C_{D}$ is the concentration of each drug, $C R$ is the ruxolitinib concentration, and is the concentration of a drug in the presence of another drug that causes a certain effect. A CI $<0.8$ indicates synergism.

Shapiro-Wilk and Levene's robust test statistic were applied to evaluate normality and homoscedasticity, respectively. Linear 
A

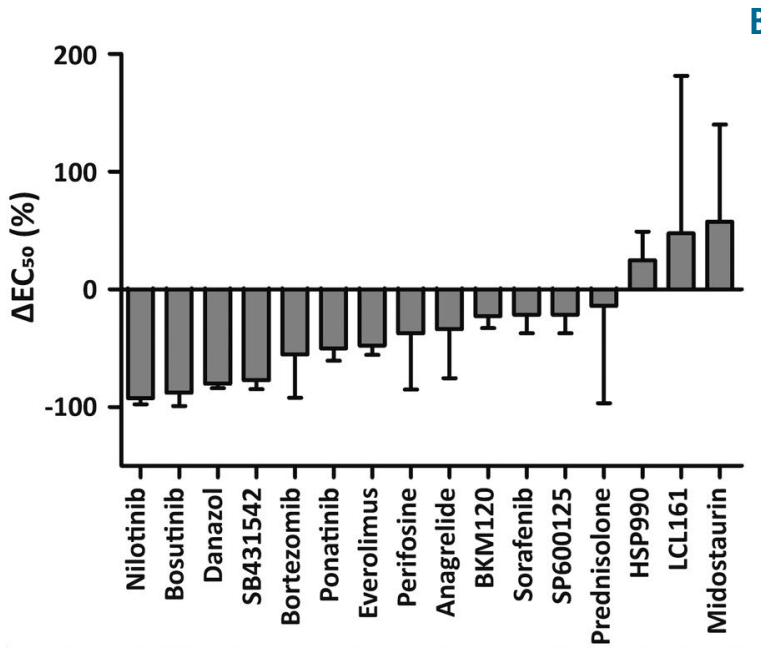

B

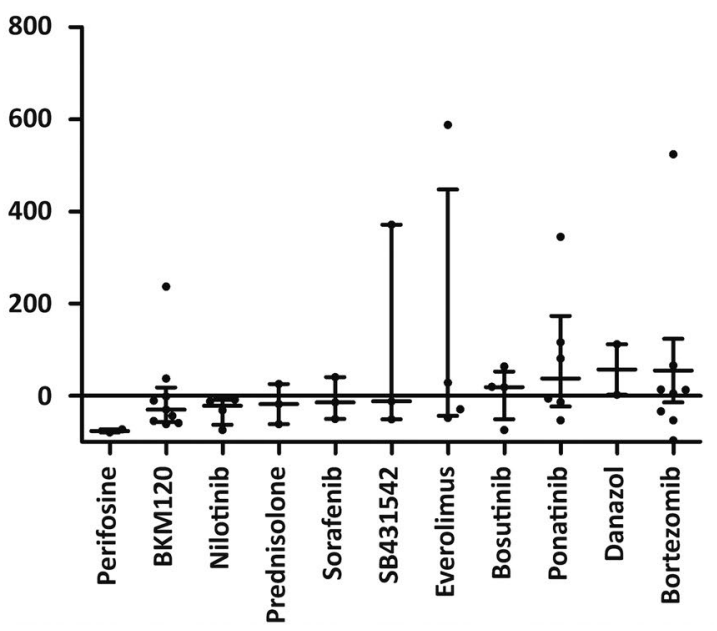

Figure 1. Effect of each drug in combination with $100 \mathrm{nM}$ ruxolitinib in BA/F3 JAK2-V617F3 cell line (A) or in patients' peripheral blood mononuclear cells (B). $\mathrm{y}$-axis: the increment between the $\mathrm{EC}_{50}$ for each drug in the presence of ruxolitinib minus its $\mathrm{EC} 50$ in monotherapy. Results are expressed as the mean \pm Standard Deviation (SD) of 2 independent experiments in cell lines (A) and median and interquartile range in patients' samples (B).

regression was performed for the correlation of time of response with ex vivo activity of ruxolitinib. For the statistical analysis of phospho-kinase array, an ANOVA test was performed. A $t$-test was used to assess whether the CI of each combination was significantly synergistic. For collagen expression assays and phosphoproteomics studies, Student $t$-test was used when the populations were normal and the non-parametric Wilcocox $t$-test when they were not. $P<0.05$ was considered statistically significant. Statistical analyses were performed with GraphPad Prism v.6.00 for Windows (GraphPad Software, La Jolla, CA, USA) or STATA v.13 (StataCorp., College Station, TX, USA).

\section{Results}

Ruxolitinib activity in cell lines and patients' samples

We first evaluated the activity of ruxolitinib in $J A K 2$-mutated cell lines. Ruxolitinib efficiently inhibited the viability of BA/F3 and SET2 V617F JAK2 cells with an $\mathrm{EC}_{50}$ of $35 \mathrm{nM}$ and $25 \mathrm{nM}$, respectively (Online Supplementary Figure $S 1 A$ ). The $\mathrm{EC}_{50}$ for ruxolitinib in BA/F3 WT cells was $212 \mathrm{nM}$, indicating the importance of the JAK2-V617F mutation for the activity of ruxolitinib. Nonetheless, when we compared the activity of ruxolitinib in patients' PBMCs with or without a JAK2 mutation, using ex vivo model $\mathrm{A}$, we found that its activity was not significantly different, with an $\mathrm{EC}_{50}$ of $55 \mathrm{nM}$. For this reason, subgroups based on the mutation in JAK2 were not studied further.

To determine the best cell model to screen drugs in combination with ruxolititinb, its activity was tested in the two different ex vivo culture models. The only method that provided a sufficient number of cells for screening was model $\mathrm{B}$, although the $\mathrm{EC}_{50}$ for ruxolititinb using this model was $0.747 \mu \mathrm{M}$. Greater ruxolitinib activity was found when PBMCs were seeded in methylcellulose in the presence of ruxolitinib (model A: $\mathrm{EC}_{50}=43 \mathrm{nM}$ ) (Online Supplementary Table $S 4$ and Online Supplementary Figure $S 1 B)$. Moreover, if ex vivo activity of ruxolitinib was com- pared with the time of response to ruxolitinib of each patient sample, it was found that both models $A$ and $B$ distinguished patients' samples with responses $>6$ months (Online Supplementary Figure S1C).

\section{BCR/ABL or ABL kinase inhibitors and PDGFR and TGF $\beta R$ inhibitors are effective combinations with ruxolitinib in cell lines and patients' samples}

To examine the best combination with ruxolitinib, dose-response curves of all tested drugs in monotherapy or in combination with ruxolitinib were first analyzed in BA/F3 V617F JAK2 cells using an automated flow cytometry platform. Drugs exhibiting the best behavior in the presence of ruxolitinib were then selected to perform the same assay using PBMCs of MF patients in ex vivo model B. Drugs with more activity in the screening were also included in dose-response assays in monotherapy with patients' samples.

Results showed that the BCR/ABL or SRC/ABL tyrosine kinase inhibitors (TKI) nilotinib and bosutinib, respectively, together with danazol, a synthetic androgen reported to reverse anemia, ${ }^{23}$ and SB432542, an inhibitor of the TGF- $\beta$ receptor related to the fibrogenic processes, were among the four best combinations in BA/F3 JAK2 V617F cells (Figure 1A). Accordingly, they presented the lowest increments between their $\mathrm{EC}_{50}$ in the presence or absence of ruxolitinib $\left(\Delta \mathrm{EC}_{50}\right.$ nilotinib $=-92.4 \% ; \Delta \mathrm{EC}_{50}$ bosutinib $=$ $-87.7 \% ; \Delta \mathrm{EC}_{50}$ danazol $=-80.1 \% ; \Delta \mathrm{EC}_{50} \mathrm{SB} 432542=$ $-77.1 \%)$. When tested in patients' samples, of the two BCR/ABL inhibitors, only nilotinib showed a lower $\mathrm{EC}_{50}$ in the presence of ruxolitinib than in its absence $\left(\Delta \mathrm{EC}_{50}\right.$ nilotinib $=-21.6 \%)$, together with SB432542 $\left(\Delta \mathrm{EC}_{50}=\right.$ $-11.7 \%$ ) (Figure 1B).

Online Supplementary Table S5 shows the drugs listed by their $\mathrm{E}_{\max }$ and $\mathrm{EC}_{50}$. The most active drugs in BA/F3 JAK2 V617F cells were the histone deacetylase HDAC6 inhibitor panobinostat $\left(\mathrm{EC}_{50}=0.041 \mu \mathrm{M}\right)$, the proteasome inhibitor bortezomib $\left(\mathrm{EC}_{50}=0.041 \mu \mathrm{M}\right)$, and the immunomodulatory HSP90 inhibitor HSP990 (EC EO $_{50}=$ 

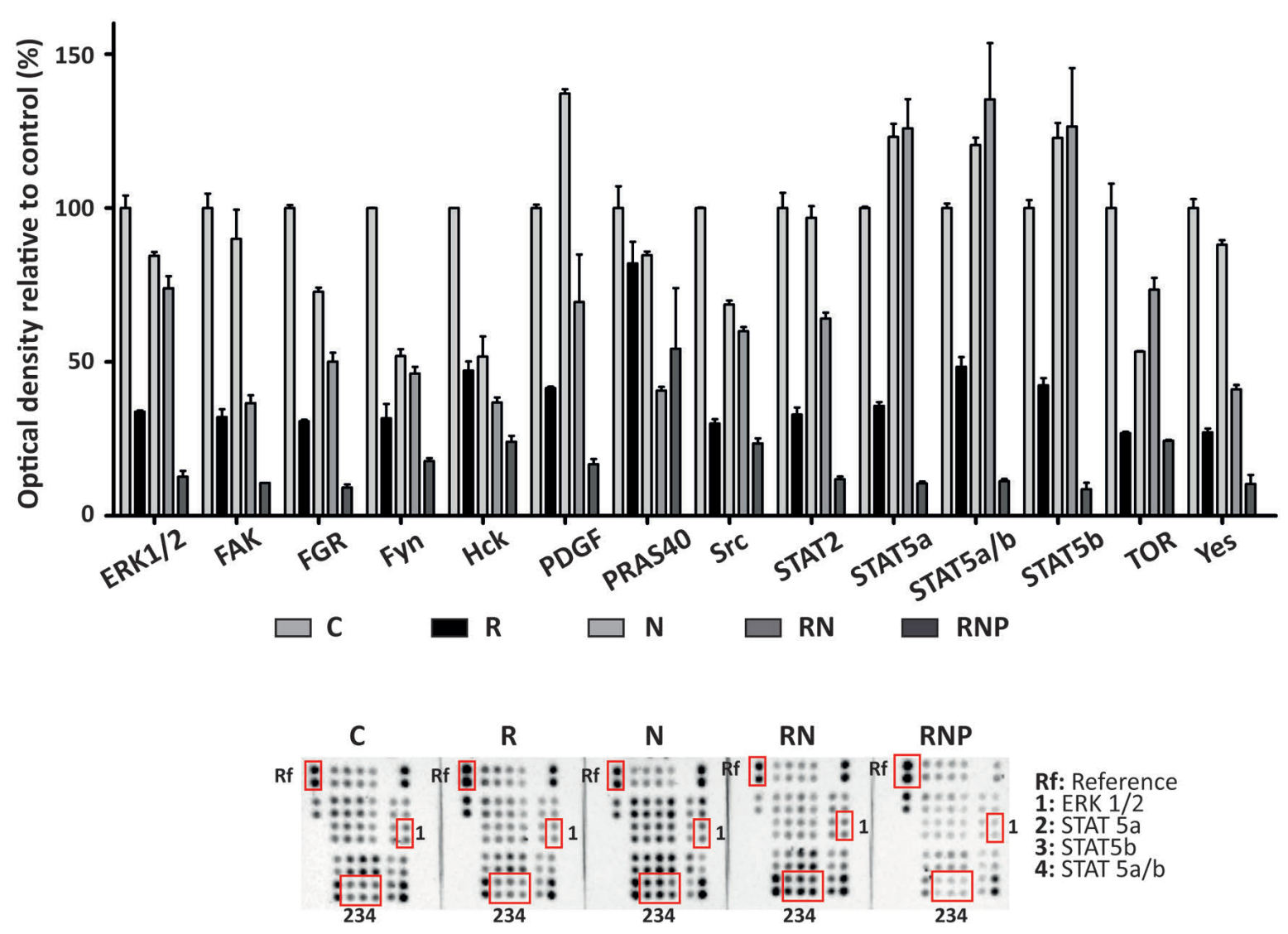

Figure 2. Screening for signaling proteins affected by $32 \mathrm{nM}$ ruxolitinib $(\mathrm{R}), 1.6 \mu \mathrm{M}$ nilotinib $(\mathrm{N}), 0.8 \mu \mathrm{M}$ prednisone $(\mathrm{P})$, and their combinations, using a phosphoprotein array. C: Control. The SET2 cell line was incubated with R, N, P or their combination for 48 hours and phosphoprotein analysis was performed.

$0.045 \mu \mathrm{M})$. Interestingly, these inhibitors were also the most active drugs in patients' samples (Table 2), showing an $\mathrm{EC}_{50}$ of $0.008 \mu \mathrm{M}, 0.033 \mu \mathrm{M}$ and $0.041 \mu \mathrm{M}$, respectively. Furthermore, prednisone, an immunosuppressant used to control symptoms of MF by decreasing the levels of cytokines and growth factors such as TGF- $\beta,{ }^{24}$ showed an $\mathrm{EC}_{50}$ of $0.144 \mu \mathrm{M}$. Other active drugs in the low micromolar range included the signaling pathway inhibitors BKM120 $\left(\mathrm{EC}_{50}=3.331 \mu \mathrm{M}\right)$ and ponatinib $\left(\mathrm{EC}_{50}=5.530\right.$ uM) (Online Supplementary Table S5). Patients' PBMCs were even more sensitive to these drugs: $\mathrm{EC}_{50}$ BKM120 = $0.893 \mu \mathrm{M}, \mathrm{EC}_{50}$ ponatinib = $1.716 \mu \mathrm{M}$ (Table 2).

Curiously, drugs used in treatment of MF such as danazol or prednisone did not have any effect on BA/F3 JAK2 V617F viability (Online Supplementary Table S5), but prednisone was effective in combination with ruxolitinib $\left(\Delta \mathrm{EC}_{50}=-13.9 \%\right)$ (Figure $\left.1 \mathrm{~A}\right)$. Patients' samples showed the same response $\left(\Delta \mathrm{EC}_{50}=-18.0 \%\right)$ (Figure $\left.1 \mathrm{~B}\right)$.

Ruxolitinib/nilotinb/prednisone combination has a synergistic effect on myeloid cell lines and patient peripheral blood mononuclear cells

Given the synergistic effect of nilotinib with ruxolitinib in BA/F3 JAK2 V617F cells, we next studied the effect of adding prednisone to this combination in myeloid cell lines and patients' PBMCs. As well as being a potent BCR/ABL TKI, nilotinib has been reported to inhibit the PDGF receptor, which is involved in fibrogenesis. ${ }^{20}$

A synergistic effect with all combinations of the three drugs tested was found in BA/F3 JAK2 V617F and SET2 cells (Table 3). By contrast, only four of the eight doses tested were synergistic in BA/F3 wild-type (WT) cells, again suggesting an important role for the V617F mutation in the activity of the drug combination. We repeated this assay using monotherapy or combination regimens in model A cultures (PBMCs) to test the effect of the combinations in myeloid progenitors. Myelofibrosis patients' samples were sensitive to nilotinib and prednisone with an EC50 of $6.6 \mu \mathrm{M}$ and $13.1 \mu \mathrm{M}$, respectively. Moreover, all combinations tested (ruxolitinib/nilotinib and ruxolitinib/niloitnib/prednisone) exhibited synergic behavior in at least two of the five patients' samples tested. In addition, the combination of $160 \mathrm{nM}$ ruxolitinib, $8 \mu \mathrm{M}$ nilotinib and $0.8 \mu \mathrm{M}$ prednisone was synergistic in all the patients' samples tested (Table 4).

\section{Ruxolitinib/nilotinb/prednisone combination blocks JAK/STAT and MAPK signaling}

The signaling pathways affected by the ruxolitinib/nilotinib/prednisone combination or monotherapy were next characterized in SET2 cells using the Proteome Profiler ${ }^{\mathrm{TM}}$ phosphoprotein array after treatment for $30 \mathrm{~min}$. Results showed that, among others, phosphorylation of STAT5 was significantly inhibited $88.94 \%$, TOR $76.55 \%$, ERK $87.55 \%$, and SRC $76.88 \%$ (Figure 2) by drug combination (ANOVA ${ }^{* * *} P<0.005$ ). Western blotting confirmed that the phosphorylation of STAT5 and ERK was inhibited by ruxolitinib in monotherapy by $69.8 \pm 8.1 \%$ and $87.7 \pm 2.3 \%$, 
respectively. In combination with nilotinib and prednisone, STAT5 and ERK were inhibited by $69.2 \pm 11.8 \%$ and $29.4 \pm 4.5 \%$, respectively (Figure $3 \mathrm{~A}$ and B). By contrast, phosphorylation of AKT was not inhibited in any case (data not shown).

\section{Ruxolitinib/nilotinib/prednisone combination decreases the synthesis of collagen I in bone marrow mesenchymal cells}

Myelofibrosis is characterized by an increase in collagen deposition, among other fibrillar proteins, in BM, which prevents its proper functioning. To study the effect of ruxolitinib, nilotinib and prednisone (in monotherapy and in combination), on collagen expression, we utilized the HS27a mesenchymal cell line, together with TGF- $\beta$ as an inductor of collagen expression. TGF- $\beta$ increased the expression of COL1A1 $200.9 \%$ over untreated cells (Figure 4A). Nilotinib decreased the mRNA expression of COL1A1 in HS27a cells by $60.2 \pm 0.9 \%$ in monotherapy, by $51.9 \pm 2.9 \%$ in combination with ruxolitinib and $62.2 \pm 1.9 \%$ in combination with ruxolitinib and prednisone. As a complementary test, we measured collagen expression by immunocytochemistry, finding that colla- gen synthesis was reduced, especially in monotherapy $(79.13 \pm 20.5 \%)$ and in combination with ruxolitinib $(79.20 \pm 2.3 \%$ ) (Figure 4B and Online Supplementary Figure S2).

\section{Discussion}

The management of MF remains challenging, even in the era of TKIs and personalized medicine. The discovery of the V617F mutation in JAK2 as a physiopathogenic mechanism of MPN3-7 prompted the development of JAK2 inhibitors and represented a revolution in the treatment of MF. Currently, the only approved JAK2 inhibitor for the treatment of MF and $\mathrm{PV}$ in the second-line is ruxolitinib, ${ }^{25}$ which has been shown to be effective in reducing hepatosplenomegaly, resolving disease-related symptoms, and producing a significant increase in overall survival when compared with conventional therapies. ${ }^{26}$ Nevertheless, there are some limitations to the use of ruxolitinib, including hematologic toxicity (anemia and thrombocytopenia) and a failure to achieve histopathological and molecular complete responses. ${ }^{15}$ Accordingly, the

Table 3. Combination Index of ruxolitinib $(\mathrm{R})$, nilotinib $(\mathrm{N})$ and prednisone $(\mathrm{P})$ in samples of cell lines. SET2: BA/F3 JAK2 wt or V617F cell lines were incubated with $\mathrm{R}, \mathrm{N}, \mathrm{P}$ or their combination for 48 hours and then Wst8 was performed.

\begin{tabular}{|c|c|c|c|c|c|c|c|c|}
\hline \multirow[b]{2}{*}{ Ruxolitinib (nM) } & \multirow[b]{2}{*}{ Nilotinib $(\mu M)$} & \multirow[b]{2}{*}{ Prednisone $(\mu \mathrm{M})$} & \multicolumn{3}{|c|}{ BAF3 JAK2 V617F } & \multicolumn{3}{|c|}{ BAF3 JAK2 wt } \\
\hline & & & CI Mean & SEM & $P$ & CI Mean & CI SEM & $\boldsymbol{P}$ \\
\hline 6.4 & 320 & & $>2$ & 9.47 & & NA & & \\
\hline 6.4 & 1.6 & & $>2$ & 3.35 & & NA & & \\
\hline 32 & 0.32 & & $>2$ & 12.49 & & NA & & \\
\hline 32 & 1.6 & & $>2$ & 187.51 & & NA & & \\
\hline 6.4 & 0.32 & 0.16 & 0.067 & 0.02 & $*$ & NA & & \\
\hline 6.4 & 0.32 & 0.8 & 0.198 & 0.12 & $* *$ & NA & & \\
\hline 6.4 & 1.6 & 0.16 & 0.082 & 0.03 & $* * *$ & 0.311 & 0.192 & \\
\hline 6.4 & 1.6 & 0.8 & 0.095 & 0.03 & $* * *$ & 0.172 & 0.090 & \\
\hline 32 & 0.32 & 0.16 & 0.049 & 0.02 & $* * *$ & $\mathrm{NA}$ & & \\
\hline 32 & 0.32 & 0.8 & 0.040 & 0.01 & $* * *$ & 0.198 & 0.086 & $* *$ \\
\hline 32 & 1.6 & 0.16 & 0.051 & 0.01 & $* * *$ & NA & & \\
\hline 32 & 1.6 & 0.8 & 0.063 & 0.01 & $* * *$ & 0.248 & 0.060 & $* *$ \\
\hline Ruxolitinib (nM) & Nilotinib $(\mu \mathrm{M})$ & Prednisone ( $\mu \mathrm{M})$ & CI Mean & $\begin{array}{l}\text { SE12 } \\
\text { SEM }\end{array}$ & $P$ & & & \\
\hline 32 & 1.6 & & 0.815 & 0.261 & & & & \\
\hline 32 & 8 & & 0.596 & 0.189 & & & & \\
\hline 160 & 1.6 & & 1.374 & 0.144 & & & & \\
\hline 160 & 8 & & 0.857 & 0.109 & & & & \\
\hline 32 & 1.6 & 0.16 & 0.538 & 0.162 & & & & \\
\hline 32 & 1.6 & 0.8 & 0.448 & 0.057 & & & & \\
\hline 32 & 8 & 0.16 & 0.396 & 0.089 & $*$ & & & \\
\hline 32 & 8 & 0.8 & 0.337 & 0.040 & $*$ & & & \\
\hline 160 & 1.6 & 0.16 & 0.155 & 0.015 & $* * *$ & & & \\
\hline 160 & 1.6 & 0.8 & 0.158 & 0.010 & $* * *$ & & & \\
\hline 160 & 8 & 0.16 & 0.173 & 0.011 & $* * *$ & & & \\
\hline 160 & 8 & 0.8 & 0.153 & 0.011 & $* * *$ & & & \\
\hline
\end{tabular}

NA: drugs combination with an effect less than of $20 \%$ compared to control. Combination Index (CI) $<0.9$ indicates synergy (Italic); CI $>1.1$ indicates antagonism (Italic Bold); CI from 0.9 to 1.1 indicates additivity (Bold). ${ }^{*} P<0.05 ;{ }^{*} P<0.01 ;{ }^{*}{ }^{*} P<0.001$. 
unmet clinical need to increase the effectiveness of the treatment and decrease its toxicity guides the search for combination treatments with ruxolitinib.

The main objective of this work was to evaluate the best combination of drugs for the treatment of MF, maintaining ruxolitinib as a therapeutic base and reducing its toxicity while maintaining its efficacy. Since MF is not characterized by large amounts of pathological cells, as in acute myeloid leukemia, ${ }^{27}$ it is challenging to develop an ex vivo model to screen 17 combinations. Consequently, we elected to utilize myeloid cells obtained from 14-day old cultures of PBMCs in methylcellulose (ex vivo model $\mathrm{B}$ ), which produce a sufficient supply of cells for screening. Interestingly, the most active drugs in monotherapy coincided with the most active drugs in preclinical trials, including bortezomib, ${ }^{28}$ panobinostat ${ }^{29}$ and HSP990. ${ }^{30}$ Therefore, the search for combination treatments with ruxolitinib is in response to

A
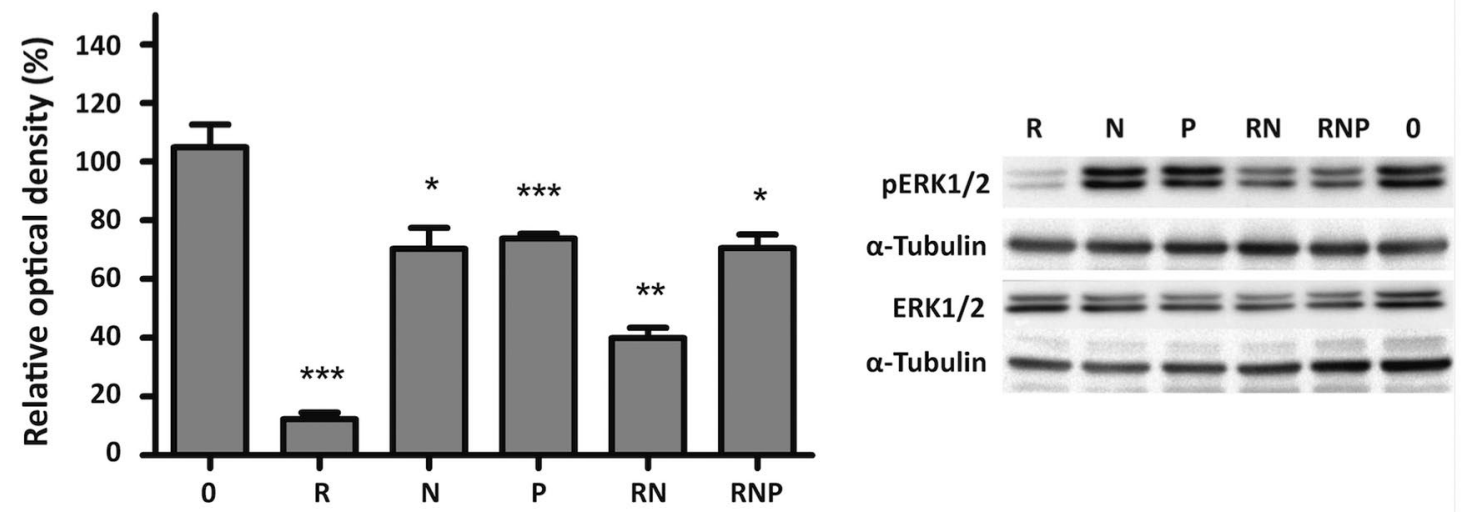

B
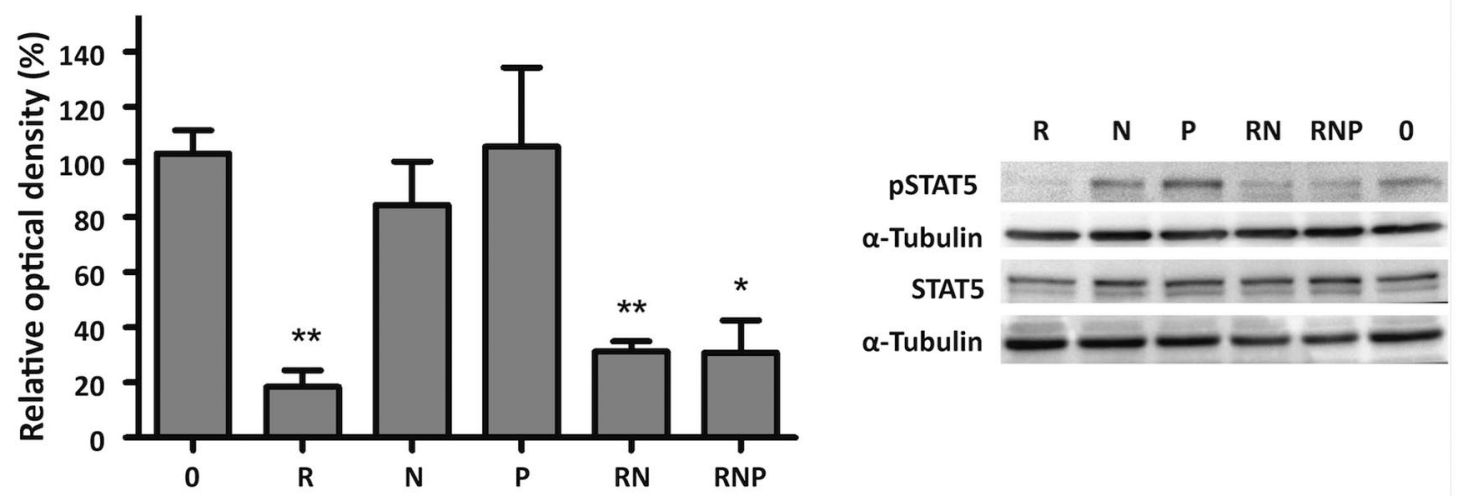

Figure 3. Effect of $32 \mathrm{nM}$ ruxolitinib (R), $1.6 \mu \mathrm{M}$ nilotinib $(\mathrm{N}), 0.8 \mu \mathrm{M}$ prednisone $(\mathrm{P})$, and their combinations, on the phosphorylation of ERK1/2 and STAT5 in cell lines. Results are expressed as the mean \pm Standard Error of Mean. Data are representative of at least 2 separate experiments. $* P<0.05 ; * * P<0.01 ; * * * P<0.001$. C: control.

Table 4. Combination Index $(\mathrm{Cl})$ of ruxolitinib $(\mathrm{R})$, nilotinib $(\mathrm{N})$ and prednisone $(\mathrm{P})$ in samples of myelofibrosis (MF) patients and healthy donors. Mononuclear cells from peripheral blood of MF patients and healthy donors were seeded at 200,000-500,000 cell/ $\mathrm{mL}$ in Metocult supplemented with SCF and IL3 in presence of drugs for two weeks. Then flow cytometry analysis was performed.

\begin{tabular}{|c|c|c|c|c|c|c|c|c|c|}
\hline Drugss & & & & & MF & & & ealthy & \\
\hline Ruxolitinib (nM) & Nilotinib ( $\mu \mathrm{M})$ & Prednisone ( $\mu \mathrm{M})$ & P20 & P33 & P27 & P19 & P34 & C1 & C3 \\
\hline 32 & 1.6 & & $>2$ & 0.50 & 1.09 & 0.40 & 0.22 & 0.24 & 0.20 \\
\hline 32 & 8 & & 1.44 & 0.18 & 0.30 & 0.34 & 0.55 & 0.12 & 0.13 \\
\hline 160 & 1.6 & & 1.83 & 1.05 & 0.32 & 0.20 & 0.16 & 0.30 & $>2$ \\
\hline 160 & 8 & & 1.55 & 0.4 & 0.22 & 0.20 & 0.05 & 0.21 & 0.13 \\
\hline 32 & 1.6 & 0.8 & $>2$ & $>2$ & 0.36 & 0.43 & 0.10 & $>2$ & $>2$ \\
\hline 32 & 1.6 & 4 & $>2$ & $>2$ & 0.15 & 0.24 & - & 0.53 & $>2$ \\
\hline 160 & 8 & 0.8 & 0.31 & 0.64 & 0.20 & 0.10 & 0.02 & 0.48 & $>2$ \\
\hline 160 & 8 & 4 & 0.39 & $>2$ & 0.13 & 0.18 & - & 0.54 & $>2$ \\
\hline
\end{tabular}

$\mathrm{CI}<0.9$ indicates sinergy (Italic); $\mathrm{CI}>\mathrm{n} 1.1$ indicates antagonism (Italic Bold). $P<0.05$ is considered significative. 
A

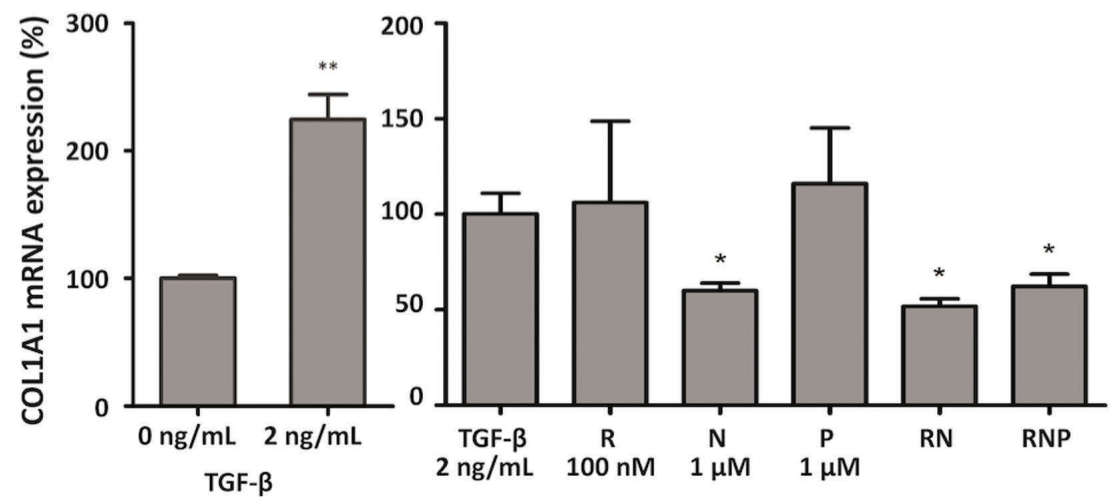

B

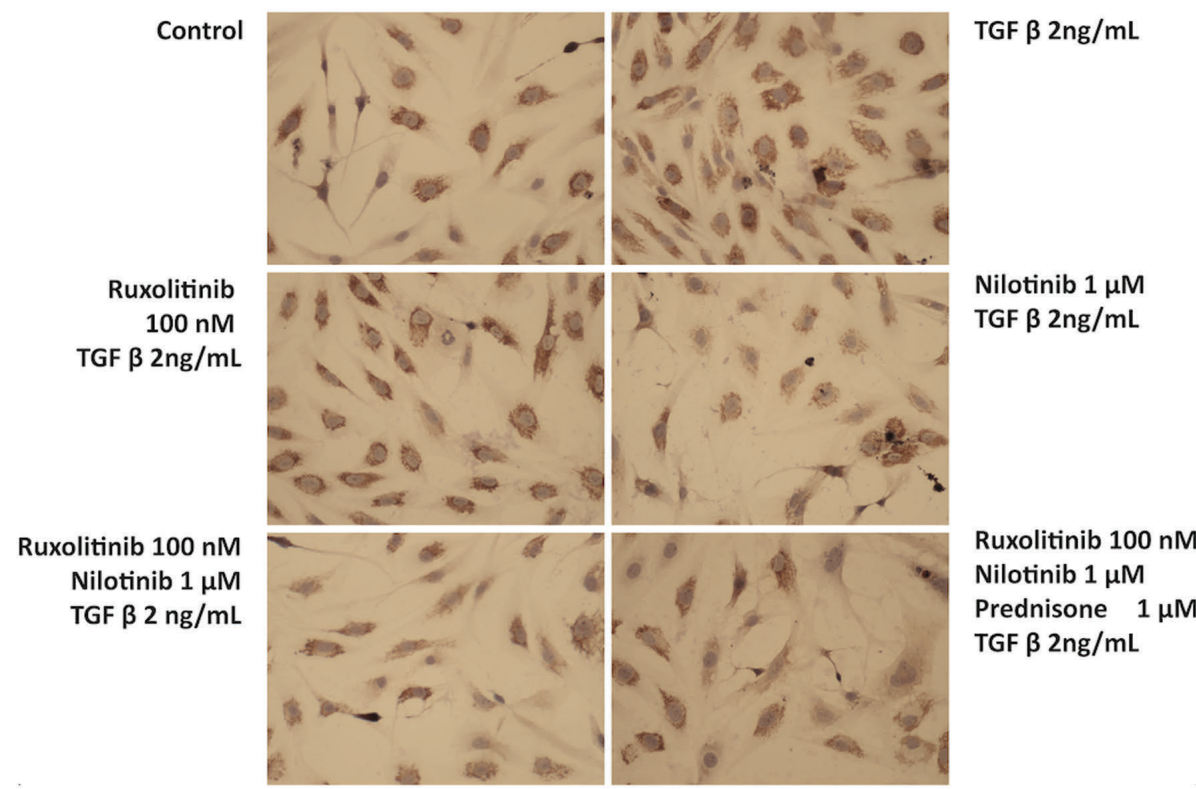

Figure 4. Effect of ruxolitinib $(\mathrm{R})$, nilotinib $(\mathrm{N})$, prednisone $(\mathrm{P})$, and their combination, on collagen mRNA expression using quantitative polymerase chain reaction or protein expression by immunocytochemistry. HS27a cell line was incubated with $\mathrm{R}, \mathrm{N}, \mathrm{P}$ or their combination for 1 hour (h) and then for $24 \mathrm{~h}$ with $2 \mathrm{ng} / \mathrm{mL}$ TGF- $\beta$. Results were expressed as the mean \pm Standard Error of Mean. Data are representative of at least 2 independent experiments. $* P<0.05 ; * * P<0.01$.

the need to increase the effectiveness of the treatment and decrease its toxicity.

We found that the best combinations were those with BCR/ABL inhibitors, nilotinib and bosutinib, used for the treatment of chronic myeloid leukemia (CML). ${ }^{31}$ Nevertheless, TKIs such as perifosine or BKM120, corticosteroids such as prednisone, androgens such as danazol, and the TGF- $\beta$ receptor inhibitor SB431542, also decreased the $\mathrm{EC}_{50}$ in combination with ruxolitinib. It is interesting to note that some of these combinations have already been tested in clinical trials, such as ruxolitinib plus danazol (clinicaltrials.gov identifier: 01732445); ${ }^{18}$ this study showed that while there was no improvement in hematologic response, stabilization of the patients was achieved. Regarding ruxolitinib plus BKM120 (clinicaltrials.gov identifier: 01730248), although no results have yet been reported, preliminary analyses (ASH 2015) are not encouraging.

Interestingly, it has previously been described that the combination nilotinib plus ruxolitinib can eliminate CD34 ${ }^{+}$ leukemic progenitors in $\mathrm{CML}^{32}$ and Philadelphia chromo- some positive acute lymphoblastic leukemia; ${ }^{33}$ however, it was not known whether this also holds for MF. We show here synergistic behavior of nilotinib plus ruxolitinib, which blocks colony formation in clonogenic assays with patients' PMBCs, and inhibits the phosphorylation of both STAT5 and ERK 1/2. Furthermore, the inclusion of prednisone is key to achieve synergy in the survival assays of cell lines and increases the power of synergy in patients' samples. In addition, this combination inhibited the synthesis of collagen in BM mesenchymal cells, which is important to achieve histopathological response. The antifibrogenic effect of nilotinib has been previously described in skin cells, ${ }^{20,34}$ liver $^{35}$ and muscle, ${ }^{36}$ via its ability to inhibit the PDGF receptor, which is directly involved in the induction of collagen synthesis. In addition, ruxolitinib is able to stabilize, or even ameliorate, fibrosis through its ability to reduce the proinflamatory state, which is typical in $\mathrm{MF}^{37}$ Similar results are seen with corticosteroids like prednisone, which decreases the levels of cytokines and proinflammatory growth factors including TGF- $\beta{ }^{24}$ 
Our results indicate that the combination ruxolitinib, nilotinib and prednisone would eliminate more efficiently pathological cells, stopping and/or reverting MF. It must be remembered, however, that this evidence was obtained in vitro and the impact on the clinical situation still needs to be proved. With this is mind, and given that all the drugs are approved for clinical practice, we have recently initiated a clinical trial (the RuNic Trial; clinicaltrials.gov identifier: 02973711) which does not require an in vivo analysis in animal models.

In summary, MF is a complex disease in which alterations in tyrosine kinase-related signaling participates in the amplification of hematopoietic clones and in the increased production of cytokines and growth factors by pathological cell clones, which stimulates $\mathrm{MF}^{38}$ It is often advantageous to use combinations of drugs that target different pathways involved in the pathophysiology of a disease, shown here with the objective of decreasing fibrosis of the BM, and not only eradicating the tumor clone, as previously attempted..$^{15,30,39,40}$ Our results lead us to hypothesize that the combination therapy ruxolitinib and prednisone might provide a dampened proinflammatory environment and nilotinib would block fibrosis. Accordingly, the combination ruxolitinib/nilotinib/prednisone is configured as a therapeutic strategy against $M F$ that aims to enhance the effect of ruxolitinib, and promote the reduction of fibrosis in the BM and reduce inflammation. As mentioned above, this combination will be studied in a phase Ib/II clinical trial in MF (the RuNic Trial; clinicaltrials.gov identifier: 02973711).

Further information is available in the Online Supplementary Appendix.

\section{Funding}

This study was supported by the Subdireccion General de Investigación Sanitaria (Instituto de Salud Carlos III, Spain) grants PI13/02387 and PI16/01530, and the CRIS against Cancer foundation grant 2014/0120. M.L. holds a postdoctoral fellowship of the Spanish Ministry of Economy and Competitiveness (FPDI-2013-16409).

\section{Acknowledgments}

The authors would like to thank to Carmen Delgado, from H12O Hematology Department for the support with the samples, Kenneth McCreath for language support, and to all patients who participated in the study.

\section{References}

1. Tefferi A, Vardiman JW. Classification and diagnosis of myeloproliferative neoplasms: The 2008 World Health Organization criteria and point-of-care diagnostic algorithms. Leukemia. 2008;22(1):14-22.

2. Tefferi A. How I treat myelofibrosis. Blood. 2011;117(13):3494-3504

3. James C, Ugo V, Le Couédic J-P, et al. A unique clonal JAK2 mutation leading to constitutive signalling causes polycythaemia vera. Nature. 2005; 434(7037):1144-1148.

4. Levine RL, Wadleigh M, Cools J, et al. Activating mutation in the tyrosine kinase JAK2 in polycythemia vera, essential thrombocythemia, and myeloid metaplasia with myelofibrosis. Cancer Cell. 2005; 7(4):387-397

5. Baxter EJ, Scott LM, Campbell PJ, et al. Acquired mutation of the tyrosine kinase JAK2 in human myeloproliferative disorders. Lancet. 2005;365(9464):1054-1061.

6. Zhao R, Xing S, Li Z, et al. Identification of an acquired JAK2 mutation in polycythemia vera. J Biol Chem. 2005;280(24):22788-22792.

7. Kralovics R, Passamonti F, Buser AS, et al. A gain-of-function mutation of JAK2 in myeloproliferative disorders. N Engl J Med. 2005;352(17):1779-1790

8. Vainchenker W, Kralovics R. Genetic basis and molecular pathophysiology of classical myeloproliferative neoplasms. Blood. 2017;129(6):667-679.

9. Ma W, Zhang X, Wang X, et al. MPL mutation profile in JAK2 mutation-negative patients with myeloproliferative disorders. Diagn Mol Pathol. 2011;20(1):34-39.

10. Klampfl T, Gisslinger H, Harutyunyan AS, et al. Somatic mutations of calreticulin in myeloproliferative neoplasms. N Engl J Med. 2013;369(25):2379-2390.

11. Nangalia J, Massie CE, Baxter EJ, et al. Somatic CALR mutations in myeloproliferative neoplasms with nonmutated JAK2. N Engl J Med. 2013;369(25):2391-2405.
12. Ha J-S, Kim Y-K. Calreticulin exon 9 mutations in myeloproliferative neoplasms. Ann Lab Med. 2015;35(1):22-27

13. Marty $\mathrm{C}$, Pecquet $\mathrm{C}$, Nivarthi $\mathrm{H}$, et al. Calreticulin mutants in mice induce an MPL-dependent thrombocytosis with frequent progression to myelofibrosis. Blood. 2016;127(10):1317-1324.

14. Chachoua I, Pecquet C, El-Khoury M, et al. Thrombopoietin receptor activation by myeloproliferative neoplasm associated calreticulin mutants. Blood. 2016; 127(10):1325-1335

15. Verstovsek S, Gotlib J, Mesa RA, et al. Long-term survival in patients treated with ruxolitinib for myelofibrosis: COMFORT-I and -II pooled analyses. J Hematol Oncol. 2017;10(1):156

16. Verstovsek S, Savona MR, Mesa RA, et al. A phase 2 study of simtuzumab in patients with primary, post-polycythaemia vera or post-essential thrombocythaemia myelofibrosis. Br J Haematol. 2017;176(6):939-949.

17. Daver N, Cortes J, Newberry K, et al. Ruxolitinib in combination with Lenalidomide as therapy for patients with myelofibrosis. Haematologica 2015; 100(8):1058-1063.

18. Gowin $\mathrm{K}$, Kosiorek H, Dueck A, et al. Multicenter phase 2 study of combination therapy with ruxolitinib and danazol in patients with myelofibrosis. Leuk Res 2017;6031-6035

19. Mascarenhas J, Hoffman R. A comprehensive review and analysis of the effect of ruxolitinib therapy on the survival of patients with myelofibrosis. Blood. 2013; 121(24):4832-4837

20. Akhmetshina A, Dees C, Pileckyte M, et al. Dual inhibition of c-abl and PDGF receptor signaling by dasatinib and nilotinib for the treatment of dermal fibrosis. FASEB J. 2008;22(7):2214-2222.

21. Arber DA, Orazi A, Hasserjian R, et al. The 2016 revision to the World Health Organization classification of myeloid neoplasms and acute leukemia. Blood. 2016; 127(20):2391-2405.
22. Chou T-C. Theoretical Basis, Experimental Design, and Computerized Simulation of Synergism and Antagonism in Drug Combination Studies. Pharmacol Rev. 2006;58(3):621-681.

23. Cervantes F, Isola IM, Alvarez-Larrán A, Hernández-Boluda J-C, Correa J-G, Pereira A. Danazol therapy for the anemia of myelofibrosis: assessment of efficacy with current criteria of response and long-term results. Ann Hematol. 2015;94(11):17911796.

24. Yu W, Guo F, Song X. Effects and mechanisms of pirfenidone, prednisone and acetylcysteine on pulmonary fibrosis in rat idiopathic pulmonary fibrosis models. Pharm Biol. 2017;55(1):450-455

25. Vainchenker W, Leroy E, Gilles L, Marty C, Plo I, Constantinescu SN. JAK inhibitors for the treatment of myeloproliferative neoplasms and other disorders. F1000Res. 2018;7:82.

26. Vannucchi AM, Kantarjian HM, Kiladjian JJ, et al. A pooled analysis of overall survival in COMFORT-I and COMFORT-II, 2 randomized phase III trials of ruxolitinib for the treatment of myelofibrosis Haematologica. 2015;100(9):1139-1145.

27. Bennett TA, Montesinos P, Moscardo F, et al. Pharmacological Profiles of Acute Myeloid Leukemia Treatments in Patient Samples by Automated Flow Cytometry: A Bridge to Individualized Medicine. Clin Lymphoma Myeloma Leuk. 2014; 14(4):305-318.

28. Wagner-Ballon $O$, Pisani DF, Gastinne $T$, et al. Proteasome inhibitor bortezomib impairs both myelofibrosis and osteosclerosis induced by high thrombopoietin levels in mice. Blood. 2007;110(1):345-353.

29. Evrot E, Ebel N, Romanet V, et al. JAK1/2 and Pan-deacetylase inhibitor combination therapy yields improved efficacy in preclinical mouse models of JAK2V617F-driven disease. Clin Cancer Res. 2013; 19(22):6230-6241.

30. Marubayashi S, Koppikar P, Taldone T, et al. HSP90 is a therapeutic target in JAK2- 
dependent myeloproliferative neoplasms in mice and humans. J Clin Invest. 2010; 120(10):3578-3593.

31. Green MR, Newton MD, Fancher KM. Off-Target Effects of BCR-ABL and JAK2 Inhibitors. Am J Clin Oncol. 2016;39(1): 76-84.

32. Gallipoli P, Cook A, Rhodes S, et al. JAK2/STAT5 inhibition by nilotinib with ruxolitinib contributes to the elimination of CML CD34+ cells in vitro and in vivo. Blood.2014;124(9):1492-1501.

33. Kong Y, Wu Y-L, Song Y, et al. Ruxolitinib/nilotinib cotreatment inhibits leukemia-propagating cells in Philadelphia chromosome-positive ALL. J Transl Med. 2017;15(1):184.

34. Luchetti MM, Moroncini G, Jose Escamez
$M$, et al. Induction of Scleroderma Fibrosis in Skin-Humanized Mice by Administration of Anti-Platelet-Derived Growth Factor Receptor Agonistic Autoantibodies. Arthritis Rheumatol. 2016; 68(9):2263-2273.

35. Liu Y, Wang Z, Kwong SO, et al. Inhibition of PDGF, TGF- $\beta$, and Abl signaling and reduction of liver fibrosis by the small molecule Bcr-Abl tyrosine kinase antagonist Nilotinib. J Hepatol. 2011;55(3):612-625.

36. Lemos DR, Babaeijandaghi F, Low M, et al. Nilotinib reduces muscle fibrosis in chronic muscle injury by promoting TNF-mediated apoptosis of fibro/adipogenic progenitors. Nat Med. 2015;21(7):786-794.

37. Massaro F, Molica M, Breccia M. How ruxolitinib modified the outcome in myelofi- brosis: focus on overall survival, allele burden reduction and fibrosis changes. Expert Rev Hematol. 2017;10(2):155-159.

38. Lataillade J-J, Pierre-Louis O, Hasselbalch $\mathrm{HC}$, et al. Does primary myelofibrosis involve a defective stem cell niche? From concept to evidence. Blood. 2008;112(8): 3026-3035.

39. Barosi G, Gattoni E, Guglielmelli P, et al Phase I/II study of single-agent bortezomib for the treatment of patients with myelofibrosis. Clinical and biological effects of proteasome inhibition. Am J Hematol. 2010;85(8):616-619

40. Tefferi A, Lasho TL, Begna KH, et al. A Pilot Study of the Telomerase Inhibitor Imetelstat for Myelofibrosis. N Engl J Med. 2015;373(10):908-919. 